\title{
INTERACTIONS BETWEEN AIRFLOW AND VALLEY TOPOGRAPHY WITH IMPLICATIONS FOR AEOLIAN SEDIMENT TRANSPORT
}

\author{
G. F. S. Wiggs \\ Sheffield Centre for International Drylands Research \\ Department of Geography, University of Sheffield \\ Western Bank, Sheffield S10 2TN \\ United Kingdom
}

\author{
J. E. Bullard \\ Department of Geography \\ Loughborough University \\ Leicestershire LE11 3TU \\ United Kingdom
}

\author{
B. Garvey \\ Sheffield Centre for International Drylands Research \\ Department of Geography, University of Sheffield \\ Western Bank, Sheffield S10 2TN \\ United Kingdom
}

\author{
I. Castro \\ School of Engineering Sciences, University of Southampton \\ Highfield, Southampton SO17 1BJ \\ United Kingdom
}

\begin{abstract}
The local topography of a landscape can have a profound influence on airflow characteristics and cause modifications to broader synoptic scale winds. This paper reports the results of a preliminary field study examining the effects of a valley on wind velocity and direction. Anemometers and wind vanes were used to measure airflow characteristics upwind, within and downwind of a 20 m deep and $175 \mathrm{~m}$ wide dry valley in the central Namib desert. The field data indicate an upwind region of flow acceleration, a minimum in flow velocity in the center of the valley, flow acceleration toward a maximum at the downwind valley edge and subsequent deceleration toward starting velocities downwind of this edge. The development of a flow separation region at the leading edge of the valley and the range of flow distortion are affected by the incident angle of the approaching wind to the axis of the valley. A conceptual model indicating the potential implications of these findings for aeolian sediment transport processes in the vicinity of dryland valleys is presented. [Key words: airflow, topography, sediment transport.]
\end{abstract}

\section{INTRODUCTION}

The local topography of a landscape can have a profound influence on airflow characteristics and cause modifications to broader synoptic scale winds. The 
impact of hilly terrain on wind speed and direction has been particularly welldocumented (e.g., Jackson and Hunt, 1975; Mason, 1987; Hunt et al., 1988; Finnigan et al., 1990; Kim et al., 2000) and there are increasing numbers of studies investigating the effects of valleys on wind regime (e.g., Whiteman and Doran, 1993; Guardans and Palomino, 1995; Sierputowski et al., 1995; Whiteman et al., 1999; Bullard et al., 2000). These studies have implications for understanding local meteorological patterns (Weber and Kaufmann, 1998), pollutant accumulation and dispersal (Beniston et al., 1989; Kalthoff et al., 2000), the intensity of aeolian processes and the distribution of aeolian sediment (Goossens and Offer, 1997; Bullard and Nash, 1998; Bullard et al., 2000). This latter focus of recent investigations is of particular importance in arid and semiarid regions where the erosion, transport and deposition of windblown sediment can be influenced by the topography of river valleys. Evidence of such interaction is provided by the close association between dunes and valley margins (Bullard and Nash, 1998, 2000) and lunette dunes with groundwater derived pans (Lawson and Thomas, 2001).

The main goal of this research is to investigate the impacts of dryland valley topography on wind speed and direction in order to understand better the effects of a valley on aeolian sediment dynamics. This research complements recent wind tunnel studies investigating the influence of valley topography on wind speed and turbulence (Sierputowski et al., 1995) and wind direction (Bullard et al., 2000).

\section{STUDY AREA}

The area chosen for the research is located in central Namibia near the eastern margin of the Namib Desert (Fig. 1). This is a region of relative tectonic stability (Lancaster, 1989a) which receives, on average, 50 to $100 \mathrm{~mm}$ rainfall per year. The main rivers in the area are the ephemeral Kuiseb, which forms the northern margin of the Namib Sand Sea to the west, and the Gaub, a tributary of the Kuiseb. The valley used for the study is located at $23^{\circ} 30^{\prime} 44^{\prime \prime} \mathrm{S}, 15^{\circ} 47^{\prime} 20^{\prime \prime} \mathrm{E}$ and is a tributary of the Gaub River (Fig. 2). The valley is dry with occasional ephemeral flow and the long axis of the valley is orientated at approximately $350^{\circ}$ relative to magnetic north. This part of the Gaub drainage basin is characterized by low relative relief with the slope gently grading down toward the north. The surface of the basin is calcrete and this overlies Tsondab sandstone into which the valley has been incised (Ward, 1987).

The site used for the field work is a section $300 \mathrm{~m}$ in length. Along this length the valley width varies from $150 \mathrm{~m}$ to $175 \mathrm{~m}$ wide at the top of the slopes. The valley sides have gradients of $20^{\circ}$ to $25^{\circ}$ and the average depth of the valley is 20 to $22 \mathrm{~m}$. The landscape surrounding the valley is a very flat plateau. The mean gradient to the west is $<1: 200$ while to the east the gradient is $<1: 1200$.

The wind regime of the central Namib has been studied extensively both from a regional perspective and in terms of local significance. The dominant influence on the general circulation pattern is the South Atlantic anticyclone (Schulze, 1972) which generates southerly winds that are deflected inland as SSW to SW winds by the thermal contrast between the land and sea. These winds are strongest and most frequent in the early austral summer-September to January (Lancaster et al., 1984). 


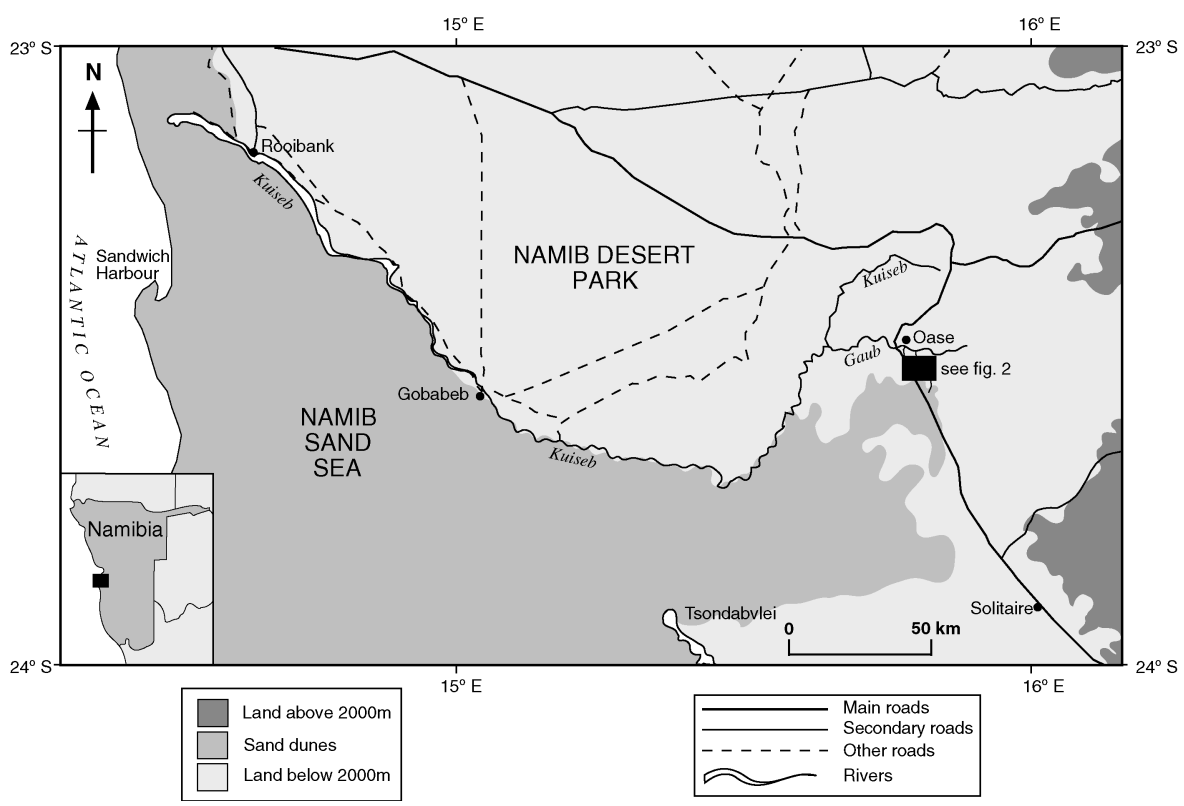

Fig. 1. Central Namibia showing the location of the study site.

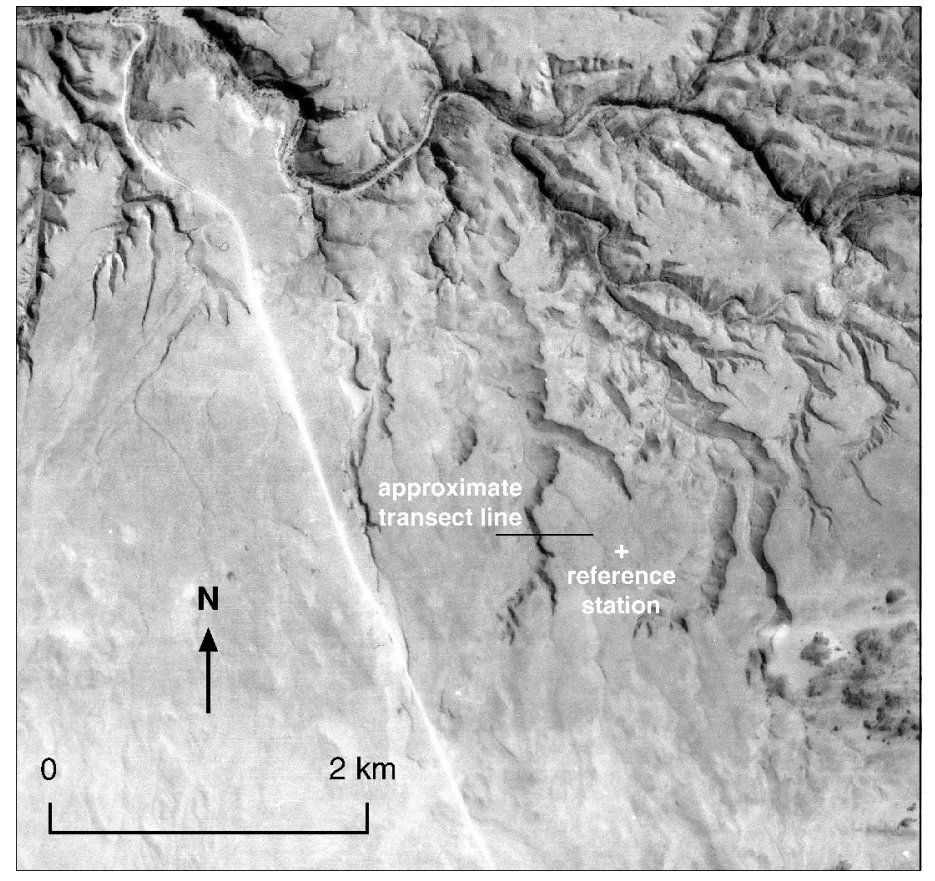

Fig. 2. Aerial photograph of the study area indicating the approximate positions of the anemometer transects and the reference station. 
The strength and geomorphological significance of these winds decrease inland (Harmse, 1982). During the austral winter the South Atlantic anticyclone weakens and the sea breezes have little influence on the inland wind regime (Lancaster, 1989a). The overall regional wind pattern is modified at the local scale by topographically and thermally driven circulation patterns (Tyson and Seely, 1980).

Lancaster (1989a) identified a three-component wind regime toward the eastern margins of the desert, where this research was conducted. This comprises the SSW to SW sea breeze, which dominates during the summer and has minimum influence during the winter, a N-NNW topographically driven plain-mountain wind common on summer mornings, and an E-ENE mountain-plain wind common on winter mornings. The latter component dominates the winter wind regime. In addition, low frequency, high magnitude "berg" winds from NE to ENE can occur. These last for periods of a few days and occur when the regional pressure gradient is normal to the coastline (Lancaster, 1989a). The berg winds are the highest velocity winds in the region and are also associated with high temperatures (Wilkinson, 1988).

This research was conducted in the winter and early spring between July and October 2000. Tyson and Seely (1980) described a daily pattern of wind direction and velocity for inland areas over the winter period in which mornings are characterized by light to moderate E to NE winds followed by light northerly winds or a calm period until the SW to W sea breeze influence increases in the afternoon. Berg winds usually start in the early morning, increase in velocity and reach a peak during the late morning with a concomitant shift in direction from SE to ESE back to NE or ENE (Lancaster, 1989a). The valley section used for this study is orientated at $350^{\circ}$, consequently, southwest winds approach the valley at an incident angle of approximately $45^{\circ}$ and easterly berg winds blow near perpendicular to the valley axis.

\section{INSTRUMENTATION AND PROCEDURE}

Wind velocity was measured along a series of transects upwind of, within, and downwind of the valley. 24 Vector A-100R pulse-count rotating cup anemometers were positioned in six vertical arrays at heights of $0.4,0.9,1.58$, and $2.78 \mathrm{~m}$ with a Vector W-200P windvane at a height of $2.2 \mathrm{~m}$. At each site, wind speed was integrated over 15-sec. intervals for periods up to $2 \mathrm{hr}$. (depending on the consistency of wind conditions). Data were recorded on-line by Campbell CR-10X dataloggers and downloaded at the end of each measurement sequence to a computer.

Velocity data from the vertical arrays were normalized with measurements at a reference station positioned at a distance of $520 \mathrm{~m}$ to the east of the center of the valley. When westerly winds were measured, normalization was carried out with reference to the upwind anemometer array. The reference station consisted of a further array of six cup anemometers at heights of $0.4,0.9,1.58,2.78,4.05$, and 5.82 $\mathrm{m}$. An array of six electronic and self-aspirating thermistors was positioned at the same heights as the anemometers in order to measure the thermal stability of the boundary layer during measurement episodes. The reference station also included a windvane positioned at a height of $2.2 \mathrm{~m}$. Analysis of the velocity data from the 
measurement arrays consisted of calculating the fractional speed-up ratio $(\delta s)$ as defined by Jackson and Hunt (1975) and used to good effect in a number of studies involving airflow over dunes (Mulligan, 1988; Wiggs, Livingstone, Thomas, and Bullard, 1996; Wiggs, Livingstone, and Warren, 1996):

$$
\delta s=\left(u_{z}-U_{z}\right) / U_{z}
$$

where $u_{z}=$ velocity at height $z$ on the measurement array, and $U_{z}=$ velocity at height $z$ at the upwind or reference station.

In using the fractional speed-up ratio flow acceleration occurs as a fraction, so a $\delta s$ of +0.10 indicates an acceleration in velocity compared to upwind values at the same height of $10 \%$.

The thermal stability of the boundary layer at the reference station was assessed through calculation of the Richardson number $(R I)$ which describes the ratio of free to forced convection (McIntosh and Thom, 1981):

$$
R I=g(\delta \theta / \delta z) / T(\delta u / \delta z)^{2}
$$

where $g=$ acceleration due to gravity, $T=$ mean profile temperature, $\delta \theta / \delta z=$ average temperature gradient, and $\delta u / \delta z=$ average velocity gradient.

In unstable conditions, where free convection is taking place, $R I$ is negative. It is positive under stable stratified conditions and approaches zero where flow is neutral and mechanical forces dominate.

\section{EXPERIMENTAL OBSERVATIONS}

\section{Velocity Measurements}

Figure 3 shows the measured fractional speed-up ratio $(\delta s)$ at two heights across the valley when the wind was blowing perpendicular to the valley from west to east. The wind conditions at the reference station evident during the measurement of this transect consisted of a mean velocity at $2.78 \mathrm{~m}$ in height of $4.48 \mathrm{~ms}^{-1}$, and a mean Richardson number of +0.141 (i.e., neutral stability conditions). The data presented here indicate an acceleration in wind velocity toward the upwind edge of the valley with wind speeds $30 \%$ higher than upwind $(\delta s=0.30)$ at a height of $2.78 \mathrm{~m}$ and $20 \%$ higher $(\delta s=0.20)$ at $0.4 \mathrm{~m}$ height. This acceleration occurs within a region $150 \mathrm{~m}$ upwind of the valley edge. This acceleration is followed by a dramatic reduction in wind velocity into the center of the valley $(\delta s \approx-0.44)$. Following this is an acceleration up the downwind valley side reaching a maximum at the edge of the valley, where $\delta s$ is 0.81 at $0.4 \mathrm{~m}$ in height and 0.53 at $2.78 \mathrm{~m}$ in height. Velocity reduces immediately downwind of this valley edge and begins to equate to upwind values within 150 to $300 \mathrm{~m}$.

Vertical profiles of fractional speed-up ratio (referenced to the upwind anemometer array) for the same transect as shown in Figure 3 are shown in Figure 4. The profiles at the upwind edge, valley center and downwind edge clearly highlight the variation in degree of acceleration with measurement height. The data in Figure 4 indicate that flow acceleration in the region immediately upwind of the valley edge 


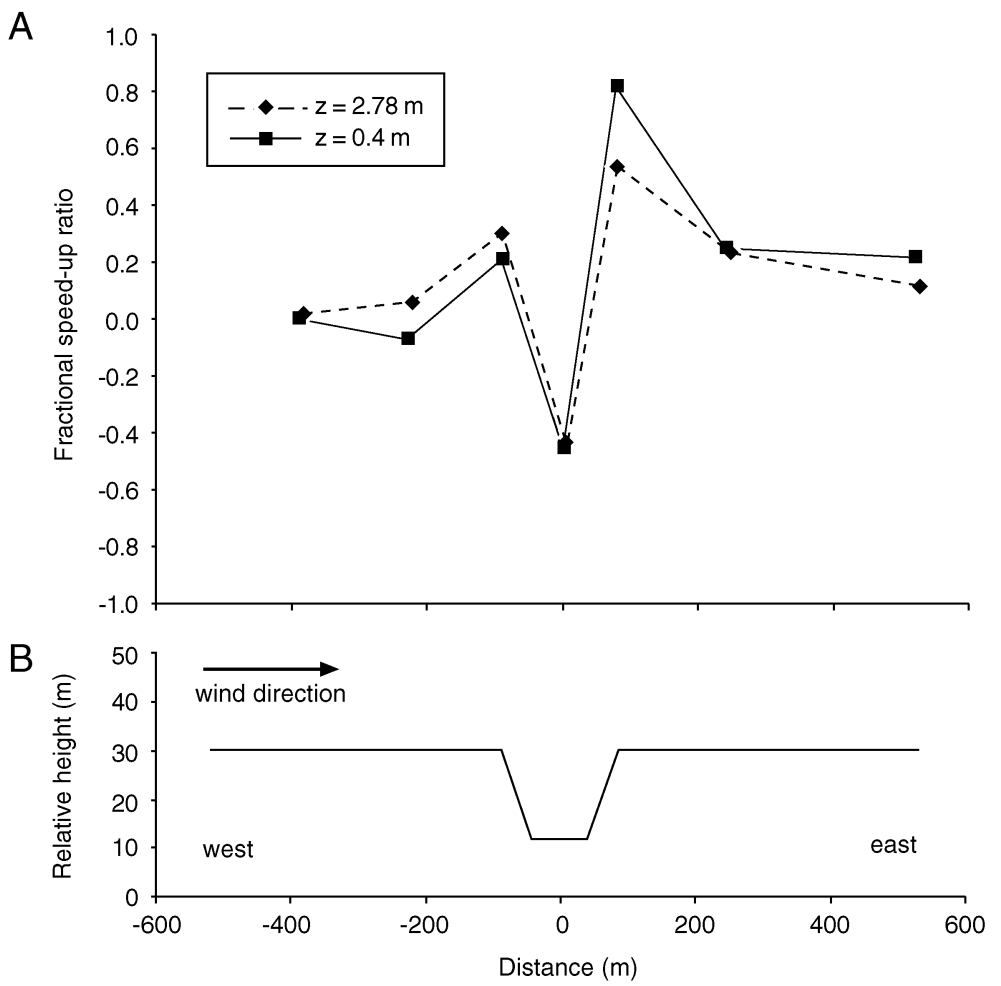

Fig. 3. (A) Fractional speed-up ratio ( $\delta$ s, relative to the upwind array) at two heights across the valley; airflow is from west to east. (B) Cross-section of the valley along the anemometer transect.

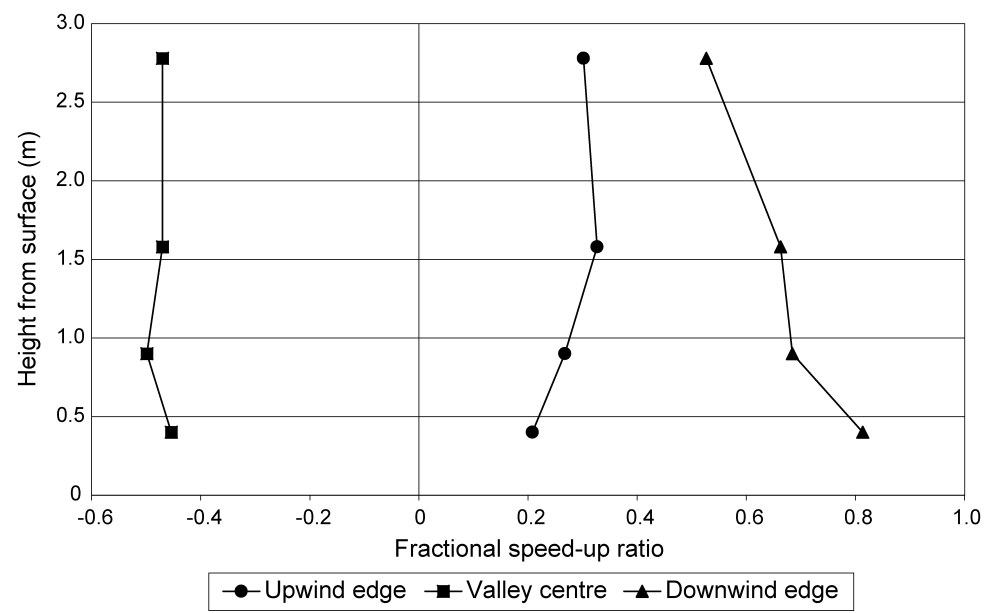

Fig. 4. Vertical profiles of fractional speed-up ratio ( $\delta$ s, relative to the upwind array) at the upwind edge, valley center and downwind edge of the valley. 
reaches a maximum at about $1.5 \mathrm{~m}$ in height. In the valley center the reduction in wind velocity is similar at all heights, while the maximum flow acceleration at the downwind valley edge is seen to be close to the surface at $0.4 \mathrm{~m}$ in height.

Similar results to those described above are evident when the wind blows at an angle to the long axis of the valley. Figure 5 shows the fractional speed-up ratio evident at two heights across the valley with a wind blowing from the NE and approaching the valley at an incident angle of approximately $37^{\circ}$. All data in this figure are referenced to velocities measured at the upwind reference station. In this transect the anemometer arrays were positioned closer together in the regions immediately upwind and downwind of the valley edges in order to gain higher resolution measurements where the maximum rate of change of velocity was evident. The wind conditions evident at the reference station during the measurement of this transect consisted of a mean velocity at $2.78 \mathrm{~m}$ in height of $4.39 \mathrm{~ms}^{-1}$, and a mean Richardson number of +0.090 (i.e., neutral stability conditions). In this example the upwind acceleration in wind velocity is less distinct and the reduction in velocity into the center of the valley also shows a reduced response. Similar to the data shown in Figure 3, the maximum acceleration for both measurement heights is evident at the downwind edge of the valley where there is acceleration from the valley floor up the downwind valley slope. Here, $\delta s$ values (relative to the reference station measurements) of 0.38 and 0.57 are evident at heights of 2.78 and $0.4 \mathrm{~m}$, respectively. These values represent a smaller degree of acceleration to those presented for the perpendicular wind (shown in Fig. 3) but also indicate maximum acceleration close to the surface.

Downwind of this peak in acceleration at the valley edge there is a reduction in wind velocity. The winds measured at the two heights presented here are shown to respond differently to this reduction. At a height of $2.78 \mathrm{~m}$, the wind velocity reduces fairly linearly with distance from $\delta s=0.38$ at the valley edge to $\delta s=0.06$ at $40 \mathrm{~m}$ downwind of the edge. Closer to the surface at a measurement height of 0.4 $\mathrm{m}$, however, the reduction in velocity is much more dramatic with a fall from $\delta s=$ 0.57 at the valley edge to $\delta s=-0.21$ within a $10 \mathrm{~m}$ distance downwind. Subsequent to this reduction these relatively near-surface winds at $0.4 \mathrm{~m}$ height increase in velocity over the following $30 \mathrm{~m}$ toward equity with upwind values.

The general pattern of windflow across the valley can be described as an increase in velocity (by up to $20 \%$ ) immediately upwind (within ca. $100 \mathrm{~m}$ ) of the valley edge and a decline in velocity of between $20 \%$ and $50 \%$ in the center of the valley. This is followed by rapid acceleration along the downwind valley slope to a maximum at the downwind valley edge with velocity increases of between $40 \%$ and $80 \%$ compared to upwind values. The windflow decelerates immediately downwind of the valley with evidence of rapid deceleration to values less than upwind close to the surface. Where such rapid near-surface deceleration is evident this is followed by acceleration of flow toward upwind velocity values. This general pattern of windflow is evident in both Figures 3 and 5, despite the data presented in each of these two transects being measured at different spatial scales (transect lengths). In comparing the two sets of data it is also apparent that the windflow pattern described above is more clearly defined and dramatic when the wind is blowing perpendicular to the valley axis. 


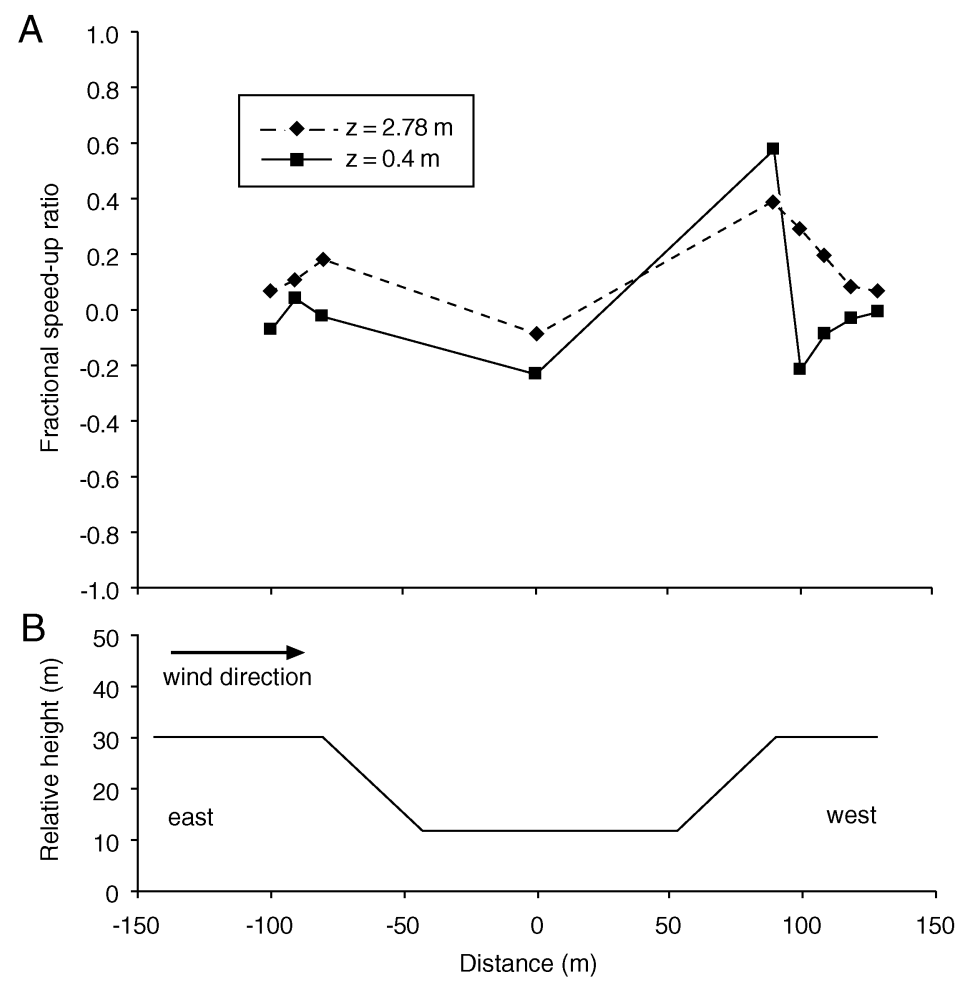

Fig. 5. (A) Fractional speed-up ratio ( $\delta$ s, relative to the upwind reference station) at two heights across the valley; airflow is from NE to SW. (B) Cross-section of the valley along the anemometer transect.

\section{Direction Measurements}

Analyses of the effect of the valley on alterations in upwind and/or downwind wind directions have so far proved inconclusive. However, the data presented in Figure 6 suggest that the direction of the within-valley winds is heavily dependent on the incident angle of the approaching wind. In this scattergraph, data are presented comparing wind direction integrated over periods of $15 \mathrm{sec}$. at the upwind edge of the valley to that measured half-way down the upwind slope. The incident angle of the approaching wind varied throughout the 2-hr. measurement period from $30^{\circ}$ to $180^{\circ}$. The data show a clustering along the $y$-axis (wind direction on slope) at around $180^{\circ}$, but with a wider scatter along this axis where the $x$-axis values (wind direction at valley edge) are less than $\sim 135^{\circ}$ (Zone A).

These data indicate that when the approach angle of the wind was greater than $\sim 135^{\circ}$ (i.e., $>45^{\circ}$ from perpendicular to the valley axis, Zone B), the wind within the valley became oriented along the direction of the valley axis (i.e., $180^{\circ}$ ). Flow visualization (using smoke flares) in the field showed that this deflection of wind direction manifested itself through flow separation at the valley edge with a 


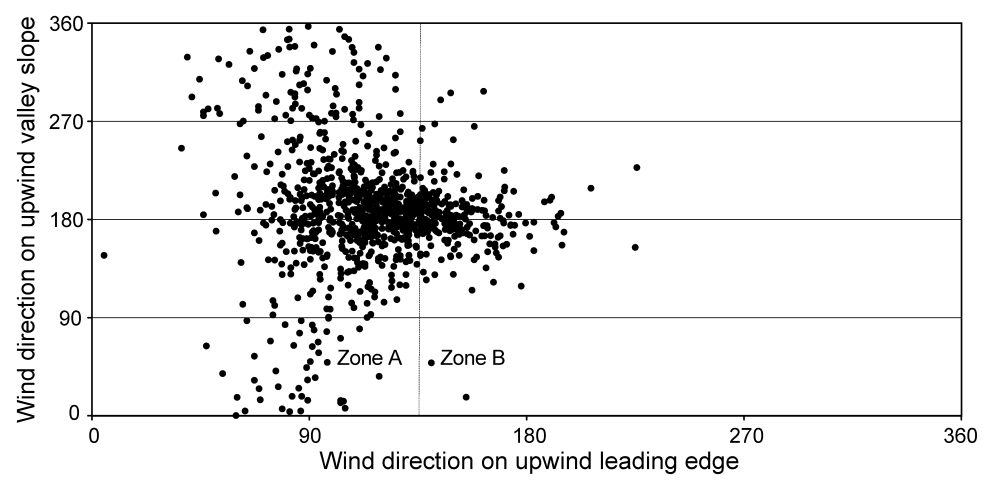

Fig. 6. A comparison of wind direction measured at the upwind edge of the valley to that measured on the upwind slope; Zone $\mathrm{A}$ = separating flow, Zone B = deflecting flow.

reattachment on the upper valley slope within about $20 \mathrm{~m}$ slope distance. The resulting small roll-vortex, which hugged the upper slopes, was characterized by a large along-valley component.

Figure 6 shows that when the approach angle of the wind was less than $135^{\circ}$ (i.e., more perpendicular to the valley axis, within $\pm 45^{\circ}$, Zone A) the resulting wind on the upwind valley slope was more variable in direction. Flow visualization showed that this wider variation in wind direction on the slope was controlled by the separation of airflow at the valley edge and the construction of a large rollvortex within the valley. Observations suggested that, when such separation occurred, the resulting large vortex feature often reattached to the surface at the center of the valley, resulting in a reversal in wind direction on the upwind valley slope and so providing measurements of wind direction here of between $225^{\circ}$ and $315^{\circ}$. In instances where no flow separation occurred at the valley edge the resulting wind direction on the upper slope was more concordant with the incident angle of the wind (i.e., between $45^{\circ}$ to $135^{\circ}$ ).

The spread of data along the $y$-axis of Figure 6 below $x$-axis values of $135^{\circ}$ is therefore explained by the irregular occurrence of airflow separation at the leading edge of the valley. Above $x$-axis values of $135^{\circ}$ the resulting wind directions on the upper valley slope tend toward conformity with the direction of the valley axis itself.

\section{DISCUSSION AND INTERPRETATION}

The field data presented in Figures 3 and 5 indicate an upwind region of flow acceleration, a minimum in flow velocity in the center of the valley, flow acceleration toward a maximum at the downwind valley edge and subsequent deceleration toward starting velocities downwind of this edge. This evolution of velocity profiles across the valley compares well with that described by Sierputowski et al. (1995) using wind tunnel experiments. Other wind tunnel investigations of airflow over forward (upwind) facing escarpments demonstrate that zones of increased surface wind velocity develop at the crest of escarpments with 
decelerating downwind flow (Bowen and Lindley, 1977). This is in accordance with the maximum in velocity at the downwind edge of the valley with subsequent downwind velocity reduction noted in the present study.

The findings described above are analogous, but essentially opposite to, wind velocities evident around positive terrain (in contrast to what could be described as negative valley terrain) such as sand dunes and hills (Taylor et al., 1987; Carruthers and Hunt, 1990; Wiggs, Livingstone, and Warren, 1996). In the case of windflow approaching hills and dunes, an adverse (increasing) pressure gradient builds up owing to the blocking effect of the mass of the hill. This results in a reduction in upwind velocities followed by a decrease in pressure up the windward slope associated with flow acceleration (and streamline compression) toward a maximum at the crest. Similar, but opposing, arguments may be applied when interpreting the upwind velocity data presented in Figures 3 and 5. As the wind approaches the leading edge of the valley it experiences a decreasing pressure gradient, leading to flow acceleration, as the airflow is drawn into the valley. Such a finding is predictable in as much as the velocity field at any point within the boundary layer is dependent on the pressure perturbations present throughout that field. So, in this case, the upwind velocity is responding to pressure perturbations caused by the valley farther downwind.

Within the valley, low wind velocities are evident in the wake region downwind of flow separation at the leading edge. This is followed by streamline compression and a negative pressure perturbation on the downwind valley slope, leading to rapid flow acceleration (in a similar manner to the windward slope of hills or dunes) toward a maximum at the downwind valley edge. This flow acceleration appears to "overshoot" at the downwind valley edge where the maximum velocities in the whole windfield are found. This overshoot in velocity at the downwind valley edge may explain the data presented in Figure 5 where dramatic flow deceleration close to the surface at this edge is followed by gradual acceleration toward upwind values. Such a rapid deceleration close to the surface at this point may be the result of near-surface flow separation at the valley edge. Downwind of this valley edge the flow recovers toward upwind values.

The direction data presented in Figure 6 reveal that winds approaching perpendicular to the valley axis are more likely to result in the creation of large roll-vortex features within the valley with little flow deflection. In contrast, winds approaching at greater incident angles develop smaller, along-valley vortex features which deflect the incoming airflow parallel to the valley axis and allow faster along-valley winds to influence the lower slopes and valley floor. These results are broadly analogous to investigations of airflow direction on sand dunes where approach flows with large incident angles result in small, along-dune vortex features developing in the lee of dune crests (e.g., Lancaster, 1989b). Similar results have also been observed in wind tunnel studies (Sierputowski et al., 1995; Bullard et al., 2000).

\section{Implications for Sediment Transport}

The association of particular sand dune types and patterns with river valleys has been widely observed in a number of desert environments, and it has been 


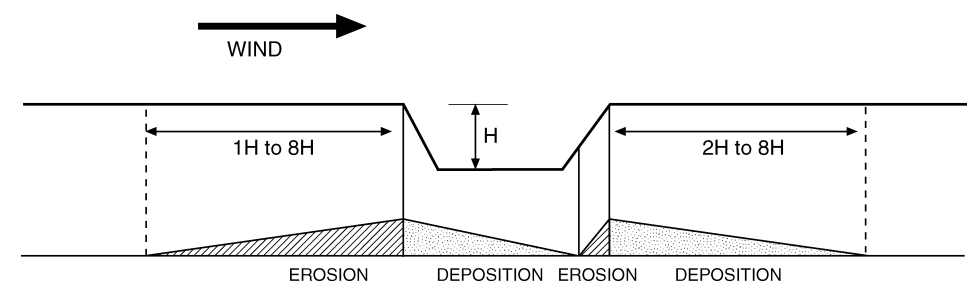

Fig. 7. A conceptual model highlighting potential zones of erosion and deposition of aeolian sediment in the vicinity of the valley assuming a perpendicular wind.

suggested that the presence of a valley may lead to modification of near-valley dune forms (e.g., Bullard and Nash, 1998, 2000). While the results presented here are of a preliminary nature, the general airflow patterns observed might have significant implications for dryland aeolian sediment transport in the vicinity of valleys.

Potential sediment transport $(q)$ is usually related to the wind by the third power of shear velocity $\left(u_{*}\right)$ :

$$
q \approx u_{*}^{3}
$$

where $u_{*}$ is proportional to the gradient of the log-normal velocity profile (Wiggs, 1997). In order to interpret the data presented here in terms of aeolian sediment transport it is therefore necessary to calculate $u_{*}$ from the measured velocity profiles. Reference to Figure 4, however, suggests that such an approach is not appropriate. Here, the acceleration of windflow with height above the surface is shown to be irregular and in these circumstances values of shear velocity $\left(u_{*}\right)$ cannot be accurately calculated from mean velocity profile data. The reason for this difficulty is that irregular flow acceleration disrupts the log-linear nature of the velocity profile, a necessity for successful $u *$ calculation (Mulligan, 1988; Wiggs, 1993; Frank and Kocurek, 1996). In order to overcome this problem the analysis presented here utilizes wind velocity close to the surface $\left(u_{z}\right)$ as a surrogate for $u_{*}$ and enables relative sediment transport potential to be considered. Such an approach is now becoming more widespread in dune investigations (Wiggs, 2001).

In considering wind velocity to be the prime control on potential aeolian sediment movement, zones of potential erosion and deposition can be determined from measurements of accelerating or decelerating flow, respectively. Assuming wind conditions similar to those presented in Figure 3, a conceptual model is presented in Figure 7 highlighting regions of potential sediment erosion and deposition in relation to the depth $(H)$ of the valley. Upwind of the valley to a distance of between $1 \mathrm{H}$ and $8 \mathrm{H}$ sediment erosion might be expected as windflow accelerates toward the valley edge. Rapid deceleration of the wind within the valley results in sediment deposition while erosion is a consequence of flow acceleration on the downwind valley slope. Immediately downwind of the valley there exists the potential for sediment deposition as the flow decelerates from a maximum velocity at the valley edge to a distance of $2 \mathrm{H}$ to $8 \mathrm{H}$. 
Assuming a ready supply of sediment and a relatively unidirectional wind regime the simple conceptual model presented in Figure 7 would suggest that dryland valleys might be characterized by: (1) an upwind region of little deposited sediment with few dunes, (2) significant within-valley aeolian sediment, and (3) a region close to the downwind valley edge of sediment deposition and dune formation.

While this conceptual model is extremely simplistic, it broadly corresponds to the spatial arrangement of some of the valley-marginal dune features described by Bullard and Nash $(1998,2000)$. There are also analogues to the deposition of lunette dunes within a short distance of the margin of pan depressions (Young and Evans, 1986; Lawson and Thomas, 2002). A further implication is that where approach winds are perpendicular to a valley axis, the valley may become a depositional sump for aeolian sediment. Conversely, the data presented in Figure 6 indicate that winds approaching at an angle to the valley axis become deflected, and so in these circumstances the valley may become a conduit for the aeolian transport of sediment along the valley axis. Such a link between the aeolian and fluvial systems in dryland regions may prove significant in terms of sediment transport and redistribution.

\section{CONCLUSION}

This study has presented a preliminary analysis of the impacts of a valley on surface airflow using field data. The results compare well with existing wind tunnel studies and suggest that, in areas of low relative relief, valley topography can have a marked impact on wind velocity and direction. The main findings of the study are:

1. An upwind region of flow acceleration is evident followed by a minimum in flow velocity in the center of the valley, flow acceleration toward a maximum at the downwind valley edge and subsequent deceleration toward starting velocities downwind of this edge. These findings are more dramatic with winds blowing perpendicular to the valley axis.

2. Winds approaching perpendicular to the valley axis are more likely to result in the creation of large roll-vortex features within the valley with little lateral flow deflection. In contrast, winds approaching at greater incident angles develop smaller, along-valley vortex features which deflect the incoming airflow parallel to the valley axis and allow faster along-valley winds to influence the lower slopes and valley floor.

A significant influence on the measurements presented here appeared to be whether or not flow separation took place at the leading edge of the valley. A major control on such separation is likely to be atmospheric stability. All the measurements presented in this study were undertaken under conditions of neutral atmospheric stability and in order to improve our understanding, future analyses will need to consider the effect of atmospheric stability on flow separation and, hence, the evolution of wind velocity and direction across the valley.

A conceptual model outlining the potential implications of valley-induced changes in the velocity profile for aeolian sediment transport has been proposed which highlights a sediment free and erosive zone upwind of the valley and a downwind sediment depositional zone. While these zones of sediment flux broadly 
correspond to some recognized aeolian dune morphologies, the model is based solely on measured velocity fluctuations. Future analysis of measurements of the turbulent Reynolds stresses upwind, within and downwind of the valley will allow significant refinement of the conceptual model.

Acknowledgments: This research was funded by a United Kingdom Natural Environment Research Council grant GR3/1210. Permission to work in the central Namib was granted by the Ministry of Environment and Tourism, Namibia (Permit No. 36060). Aerial photographs are reproduced by kind permission of the Surveyor-General's Office, Namibia. The authors would like to thank Kücki, Willi Probst, and Leilo Probst for invaluable assistance at the field site, Joh Henschel (Desert Research Foundation of Namibia) and everyone at the Desert Ecological Research Unit, Gobabeb, for their assistance and logistical support, Mark Szegner for cartography, and Rob Bryant for help with figures. We also are grateful to Vatche Tchakerian for helpful comments and support.

\section{REFERENCES}

Beniston, M., Ruffieux, D., and Hertig, J-A. (1989) A combined numerical and wind-tunnel study of ventilation and air pollution episodes in a rural valley of Switzerland. Boundary-Layer Meteorology, Vol. 48, 129-156.

Bowen, A. J. and Lindley, D. (1977) A wind-tunnel investigation of the wind speed and turbulence characteristics close to the ground over various escarpment shapes. Boundary-Layer Meteorology, Vol. 12, 259-271.

Bullard, J. E. and Nash, D. J. (1998) Linear dune pattern variability in the vicinity of dry valleys in the southwest Kalahari. Geomorphology, Vol. 23, 35-54.

Bullard, J. E. and Nash, D. J. (2000) Valley-marginal sand dunes in the south-west Kalahari: Their nature, classification and possible origins. Journal of Arid Environments, Vol. 45, 369-383.

Bullard, J. E., Wiggs, G. F. S., and Nash, D. J. (2000) Experimental study of wind directional variability in the vicinity of a model valley. Geomorphology, Vol. 35, 127-143.

Carruthers, D. J. and Hunt, J. C. R. (1990) Fluid mechanics of air flow over hills: Turbulence, fluxes and waves in the boundary layer. In R. M. Banta, ed., Atmospheric Processes over Complex Terrain, Meteorological Monographs, Volume 23. Boston, MA: American Meteorological Society, 88-103.

Finnigan, J. J., Raupach, M. R., Bradley, E. F., and Aldis, G. K. (1990) A wind tunnel study of turbulent flow over a two-dimensional ridge. Boundary-Layer Meteorology, Vol. 50, 277-317.

Frank, A. and Kocurek, G. (1996) Airflow up the stoss slope of sand dunes: Limitations of current understanding. Geomorphology, Vol. 17, 47-54.

Goossens, D. and Offer, Z. Y. (1997) Aeolian dust erosion on different types of hills in a rocky desert: Wind tunnel simulations and field measurements. Journal of Arid Environments, Vol. 37, 209-229.

Guardans, R. and Palomino, I. (1995) Description of wind field dynamic patterns in a valley and their relation to mesoscale and synoptic-scale meteorological situations. Journal of Applied Meteorology, Vol. 34, 49-67.

Harmse, J. T. (1982) Geomorphologically effective winds in the northern part of the Namib sand desert. South African Geographer, Vol. 10, 43-52. 
Hunt, J. C. R., Leibovich, S., and Richards, K. J. (1988) Turbulent shear flows over low hills. Quarterly Journal of the Royal Meteorological Society, Vol. 114, 14351470.

Jackson, P. S. and Hunt, J. C. R. (1975). Turbulent wind flow over a low hill. Quarterly Journal of the Royal Meteorological Society, Vol. 101, 929-955.

Kalthoff, N., Horlacher, V., Corsmeier, U., Volz-Thomas, A., Kolhagar, B., Geiss, H., Mollmann-Coers, M., and Knaps, A. (2000) Influence of valley winds on transport and dispersion of airborne pollutants in the Freiburg-Schauinsland area. Journal of Geophysical Research-Atmospheres, Vol. 105, 1585-1597.

Kim, H. G., Patel, V. C., and Lee, C. M. (2000) Numerical simulation of wind flow over hilly terrain. Journal of Wind Engineering and Industrial Aerodynamics, Vol. 87, 45-60.

Lancaster, J., Lancaster, N., and Seely, M. (1984) The climate of the central Namib. Madoqua, Vol. 14, 5-61.

Lancaster, N. (1989a) The Namib Sand Sea: Dune Forms, Processes and Sediments. Rotterdam, The Netherlands: Balkema.

Lancaster, N. (1989b) The dynamics of star dunes: An example from the Gran Desierto, Mexico. Sedimentology, Vol. 36, 273-289.

Lawson, M. and Thomas, D. S. G. (2001) Late Quaternary lunette dune sedimentation in the southwestern Kalahari Desert, South Africa: Luminescence based chronologies of aeolian activity. Quaternary Science Reviews, Vol. 21, 825-836.

Mason, P. J. (1987) Diurnal variations in flow over a succession of ridges and valleys. Quarterly Journal of the Royal Meteorological Society, Vol. 113, 11171140.

McIntosh, D. W. and Thom, A. S. (1981) Essentials of Meteorology. London, UK: Taylor and Francis.

Mulligan, K. R. (1988) Velocity profiles measured on the windward slope of a transverse dune. Earth Surface Processes and Landforms, Vol. 13, 573-582.

Schulze, B. R. (1972) South Africa. In J. F. Griffiths, ed., World Survey of Climatology Vol. 10, Climates of Africa. Amsterdam, The Netherlands: Elsevier.

Sierputowski, P., Ostrowski, J., and Cenedese, A. (1995) Experimental study of wind flow over the model of a valley. Journal of Wind Engineering and Industrial Aerodynamics, Vol. 57, 127-136.

Taylor, P. A., Mason, P. J., and Bradley, E. F. (1987) Boundary layer flow over low hills. Boundary-Layer Meteorology, Vol. 39, 107-132.

Tyson, P. D. and Seely, M. (1980) Local winds over the central Namib. South African Geographical Journal, Vol. 62, 136-150.

Ward, J. D. (1987) The Cenozoic Succession in the Kuiseb Valley, Central Namib Desert. Windhoek, Namibia: Geological Survey of Namibia Memoir No. 9, Department of Economic Affairs.

Weber, R. O. and Kaufmann, P. (1998) Relationship of synoptic winds and complex terrain flows during the MISTRAL field experiment. Journal of Applied Meteorology, Vol. 37, 1486-1498.

Whiteman, C. D., Bian, X. D., and Sutherland, J. L. (1999) Wintertime surface wind patterns in the Colorado River valley. Journal of Applied Meteorology, Vol. 38, 1118-1130. 
Whiteman, C. D. and Doran, J. C. (1993) The relationship between overlying synoptic-scale flows and winds within a valley. Journal of Applied Meteorology, Vol. 32, 1669-1682.

Wiggs, G. F. S. (1993) Desert dune dynamics and the evaluation of shear velocity: An integrated approach. In K. Pye, ed., The Dynamics and Environmental Context of Aeolian Sedimentary Systems. London, UK: The Geological Society of London. Special Publication 72, 37-46.

Wiggs, G. F. S. (1997) Sediment mobilisation by the wind. In D. S. G. Thomas, ed., Arid Zone Geomorphology. Chichester, UK: Wiley, 351-372.

Wiggs, G. F. S. (2001) Desert dune processes and dynamics. Progress in Physical Geography, Vol. 25, 55-81.

Wiggs, G. F. S., Livingstone, I., Thomas, D. S. G., and Bullard, J. E. (1996) Airflow and roughness characteristics over partially-vegetated linear dunes in the southwest Kalahari desert. Earth Surface Processes and Landforms, Vol. 21, 19-34.

Wiggs, G. F. S., Livingstone, I., and Warren, A. (1996) The role of streamline curvature in sand dune dynamics: Evidence from field and wind tunnel measurements. Geomorphology, Vol. 17, 29-46.

Wilkinson, M. J. (1988) Linear dunes in the central Namib desert: Theoretical and chronological perspectives from wind streaks. In G. F. Dardis and B. P. Moon, eds., Geomorphological Studies in Southern Africa. Rotterdam, The Netherlands: Balkema, 85-113.

Young, J. A. and Evans, R. A. (1986) Erosion and deposition of fine sediments from playas. Journal of Arid Environments, Vol. 10, 103-115. 